Fixed Point Theory, 21(2020), No. 2, 693-696

DOI: $10.24193 /$ fpt-ro.2020.2.48

http://www.math.ubbcluj.ro/ nodeacj/sfptcj.html

\title{
AN ULTRA-PRODUCT METHOD VIA LEFT REVERSIBLE SEMIGROUPS TO STUDY BRUCK'S GENERALIZED CONJECTURE
}

\author{
FOUAD NADERI \\ Department of Mathematical and Statistical Sciences \\ University of Alberta, Edmonton \\ Alberta, T6G 2G1, Canada \\ E-mail: naderi@ualberta.ca
}

\begin{abstract}
It has been asked by Lau several times whether a Banach space with weak fixed point property has weak fixed point property for left reversible semigroups. This problem is known as Bruck generalized conjecture (BGC). The aim of this note is to propose a new approach to tackle the BGC. Our approach uses the order structure of the semigroup for the first time in literature to construct an ultra-product structure. Then, we use this ultra-product structure to give an affirmative answer to BGC for the case of nearly uniformly convex (NUC) Banach spaces. One should note that alternatives proofs are available in the case of NUC Banach spaces, but what we hope for is that the originality of our method could pave the way for studying the BGC in its utmost generality.

Key Words and Phrases: Nearly uniformly convex Banach space, non-expansive mapping, weak
\end{abstract} fixed point property.

2010 Mathematics Subject Classification: 47H10, 54H25.

Acknowledgments. The author would like to thank Professor A. T. M. Lau for his invaluable remarks and suggestions. He also would like to thank Professor W. A. Kirk for his feedback.

\section{REFERENCES}

[1] M. Amini, A.R. Medghalchi, F. Naderi, Pointwise eventually non-expansive action of semitopological semigroups and fixed points, J. Math. Anal. Appl., 437(2016), 1176-1183.

[2] R.E. Bruck Jr., A common fixed point theorem for a commuting family of non-expansive mappings, Pacific J. Math., 53(1974), 59-71.

[3] M. Edelstein, The construction of asymptotic center with a fixed point property, Bull. Amer. Math. Soc., 78(1972), 206-208.

[4] R.D. Holmes, A.T. Lau, Asymptotically non-expansive actions of topological semigroups and fixed points, Bull. London. Math. Soc., 3(1971), 343-347.

[5] A.T.M. Lau, Normal structure and common fixed point properties for semigroups of nonexpansive mappings in Banach spaces, Fixed Point Theory Appl. 2010, Art. ID 580956, 14 pp.

[6] T.C. Lim, Characterization of normal structure, Proc. Amer. Math. Soc., 43(1974), 313-319.

[7] F. Naderi, Weak* fixed point property of reduced Fourier-Stieltjes algebra and generalization of Baggett's theorem, J. Math. Anal. Appl., 461 (2018), 451-460. 
[8] A. Wisnicki, J. Wosko, Banach ultrapowers and multivalued non-expansive mappings, J. Math. Anal. Appl., 326(2007), 845-857.

Received: October 9, 2017; Accepted: January 20, 2018. 